\title{
Focusing of sub-micrometer particles and bacteria enabled by two-dimensional acoustophoresis.
}

\author{
Antfolk, M.; Muller, Peter Barkholt; Augustsson, P.; Bruus, Henrik; Laurell, T.
}

Published in:

Lab on a Chip

Link to article, DOI:

$10.1039 / \mathrm{c} 4 \mathrm{lc00202d}$

Publication date:

2014

Document Version

Publisher's PDF, also known as Version of record

Link back to DTU Orbit

Citation (APA):

Antfolk, M., Muller, P. B., Augustsson, P., Bruus, H., \& Laurell, T. (2014). Focusing of sub-micrometer particles and bacteria enabled by two-dimensional acoustophoresis. Lab on a Chip, 14(15), 2791-2799.

https://doi.org/10.1039/c4lc00202d

\section{General rights}

Copyright and moral rights for the publications made accessible in the public portal are retained by the authors and/or other copyright owners and it is a condition of accessing publications that users recognise and abide by the legal requirements associated with these rights.

- Users may download and print one copy of any publication from the public portal for the purpose of private study or research.

- You may not further distribute the material or use it for any profit-making activity or commercial gain

- You may freely distribute the URL identifying the publication in the public portal 


\title{
Lab on a Chip
}

\section{PAPER}

Cite this: Lab Chip, 2014, 14, 2791

\section{Focusing of sub-micrometer particles and bacteria enabled by two-dimensional acoustophoresis $\dagger$}

\author{
M. Antfolk, ${ }^{* a}$ P. B. Muller, ${ }^{\text {b }}$ P. Augustsson, ${ }^{\text {ac }}$ H. Bruus ${ }^{b}$ and T. Laurell ${ }^{* a}$
}

Handling of sub-micrometer bioparticles such as bacteria are becoming increasingly important in the biomedical field and in environmental and food analysis. As a result, there is an increased need for less labor-intensive and time-consuming handling methods. Here, an acoustophoresis-based microfluidic chip that uses ultrasound to focus sub-micrometer particles and bacteria, is presented. The ability to focus sub-micrometer bioparticles in a standing one-dimensional acoustic wave is generally limited by the acoustic-streaming-induced drag force, which becomes increasingly significant the smaller the particles are. By using two-dimensional acoustic focusing, i.e. focusing of the sub-micrometer particles both horizontally and vertically in the cross section of a microchannel, the acoustic streaming velocity field can be altered to allow focusing. Here, the focusability of $E$. coli and polystyrene particles as small as $0.5 \mu \mathrm{m}$ in diameter in microchannels of square or rectangular cross sections, is demonstrated. Numerical

Received 14th February 2014, Accepted 20th May 2014

DOI: $10.1039 / c 4 l c 00202 d$

www.rsc.org/loc analysis was used to determine generic transverse particle trajectories in the channels, which revealed spiral-shaped trajectories of the sub-micrometer particles towards the center of the microchannel; this was also confirmed by experimental observations. The ability to focus and enrich bacteria and other sub-micrometer bioparticles using acoustophoresis opens the research field to new microbiological applications.

\section{Introduction}

The ability to control and process sub-micrometer bioparticles, e.g. bacteria and subcellular organelles, is becoming increasingly important in biomedicine and in environmental and food analysis. ${ }^{1,2}$ Methods such as blood culture of bacteria $^{1}$ and subcellular fractionation ${ }^{3}$ are, however, laborintensive, complicated, and time-consuming, and new technologies are being sought to redress these shortcomings.

Microfluidics offers a means of automated handling and analysis of sub-micrometer bioparticles with the associated advantage of a continuous mode of sample handling. Thus, considerations such as initial sample volume or batch volume are no longer relevant. Previously used methods for handling of sub-micrometer particles included filters, ${ }^{4,5}$ dielectrophoresis, ${ }^{6-8}$ inertia in combination with hydrodynamic forces, ${ }^{9}$ magnetophoresis, ${ }^{10,11}$ deterministic lateral displacement, ${ }^{12}$ and surface acoustic waves (SAW). ${ }^{13}$ These

\footnotetext{
${ }^{a}$ Department of Biomedical Engineering, Lund University, Box 118, SE-221 00 Lund, Sweden. E-mail: thomas.laurell@bme.lth.se, maria.antfolk@bme.lth.se ${ }^{b}$ Department of Physics, Technical University of Denmark, DTU Physics Bldg 309, DK-2800 Kongens Lyngby, Denmark

${ }^{c}$ Department of Electrical Engineering and Computer Science, Massachusetts Institute of Technology, Cambridge, MA, USA

$\dagger$ Electronic supplementary information (ESI) available. See DOI: 10.1039/ c4lc00202d
}

methods have been mainly used for handling of bacteria and particles of around $1 \mu \mathrm{m}$ in diameter. Recently, SAW were used to separate $0.5 \mu \mathrm{m}$ polystyrene particles from $0.3 \mu \mathrm{m}$ particles, ${ }^{14}$ Stoneley waves were used to focus $0.5 \mu \mathrm{m}$ polystyrene particles at flow rates of $200 \mathrm{~nL} \min ^{-1}, 15$ and acoustic trapping has been used to successfully trap $0.1 \mu \mathrm{m}$ particles using seeding particles. ${ }^{16}$ Although acoustic seed trapping gives good recovery of sub-micrometer particles and bacteria, the system operates in batch mode, which is limited by the capacity of the acoustic trap. In spite of these developments, one common need is the ability to process sub-micrometer particles in continuous-flow mode together with the possibility of handling rare species in crude samples with high recovery rates without previous sample preparation.

In this regard, the use of acoustophoresis in microfluidic systems has attracted much attention in recent years as a continuous-flow and non-contact mode method of separating or enriching microparticles or cells while offering a reasonable degree of throughput. The method involves the use of ultrasound standing waves to focus cells or particles in the nodal (or anti-nodal) plane of the standing wave according to their intrinsic properties: size, density, and compressibility. ${ }^{17}$ Furthermore, this label-free and gentle ${ }^{18,19}$ method-which operates independently of the biochemical and electrical properties of the suspension medium-has been extensively explored to separate, wash, or concentrate various biological 
samples including blood, ${ }^{20-23}$ raw milk, ${ }^{24}$ circulating tumor cells, ${ }^{25,26}$ and yeast. ${ }^{27}$

For bulk acoustic waves (BAW) microchannel acoustophoresis is usually carried out in the 1-10 $\mathrm{MHz}$ frequency range and particles are focused along a single dimension. For larger particles, the acoustically induced particle motion is dominated by the primary acoustic radiation force, whereas the motion of smaller particles is instead dominated by the acoustic streaming-induced drag force of the suspending liquid. ${ }^{28,29}$ Attempts have been made to address the need for bacterial or other sub-micrometer particle manipulation and enrichment in acoustic standing-wave systems. Bacteria have been processed with some success in batch mode using ultrasound to agglomerate them, ${ }^{30,31}$ and a quarter-wavelength acoustic device was used to concentrate $1 \mu \mathrm{m}$ particles in continuous flow. ${ }^{32}$ However, no systems have yet emerged that enable continuous flow-based focusing of bacteria or other sub-micrometer particles at recovery rates above $90 \%$, relevant when handling highly dilute suspensions.

This paper presents continuous flow-based sub-micrometer particle focusing using two-dimensional BAW-acoustophoresis. The use of two-dimensional focusing has previously only been explored for particles larger than $5 \mu \mathrm{m}$ in diameter. ${ }^{25,26,33-35}$ In contrast to the case with one-dimensional standing acoustic waves, the simultaneous excitation of two orthogonal resonances generates an acoustic streaming velocity field that does not counteract the primary radiation force. A numerical model that predicts a streaming field with essentially a single large vortex centered in the cross section of the channel, in agreement with experimental data, is also presented.

\section{Theory}

Particles in a standing-wave acoustophoresis system are primarily affected by two forces: the acoustic radiation force from scattering of the acoustic wave on the particles, and the drag force from the acoustic streaming velocity field of the fluid generated by viscous stresses in the acoustic boundary layers. The interplay between these two forces and the regimes in which they each dominate the particle motion in acoustophoresis systems have been studied extensively by Barnkob et al. ${ }^{29}$ Through theoretical derivation and experimental verification, these authors have described how the motion of large particles is dominated by the acoustic radiation force while the motion of small particles is dominated by the drag force from the acoustic streaming.

To theoretically determine the critical particle diameter $2 a_{\mathrm{c}}$, where the crossover from radiation force-dominated particle motion to acoustic streaming-induced drag forcedominated particle motion occurs, the magnitudes of the two forces are equated, resulting in the following equation valid for single-particle motion in a half-wavelength resonance: ${ }^{29}$

$$
2 a_{\mathrm{c}}=\sqrt{\frac{12 s}{\pi} \frac{v}{\Phi f}} \approx 1.6 \mu \mathrm{m},
$$

where $s$ is a factor related to the channel geometry, $v$ is the kinematic viscosity of the medium, $\Phi$ is the acoustic contrast factor, and $f$ is the frequency of the acoustic field. The numerical value is calculated for a polystyrene particle in water and a frequency of $f=3.19 \mathrm{MHz}$. The geometrical value used is $s=0.47$ for a particle near the top or bottom walls, and includes thermal effects. ${ }^{29}$ The critical particle size is independent of the applied peak-to-peak voltage $U_{\mathrm{pp}}$ driving the piezo-ceramic ultrasound transducer, because both the radiation force and the streaming depend linearly on the energy density of the standing acoustic wave. In contrast, it can be seen in eqn (1) that the critical particle size does depend on the material parameters $v$ of the fluid and $\Phi$ of the fluid and particles, and on the actuation frequency $f$. Increasing the frequency to achieve radiation forcedominated motion of smaller particles is a relatively straightforward solution, but such an increase often necessitates reduced channel dimensions, which drastically reduces the throughput of the device. In this paper we propose another solution, namely to change the whole acoustic resonance such that the acoustic radiation force and the acoustic streaming-induced drag work together in focusing the particles.

The acoustic streaming and acoustophoretic particle motion in a microchannel cross section have been studied numerically by Muller et $a l^{28}$ The method is valid for long, straight microchannels of constant rectangular cross section and employs a pertubation approach to the pressure, temperature and velocity fields. Briefly, the numerical scheme is as follows. The first-order acoustic fields are solved in the frequency domain for an oscillating velocity boundary condition on the walls of the rectangular channel domain. From the first-order fields, the acoustic radiation force is calculated from the expression given by Settnes and Bruus, ${ }^{36}$ while the steady acoustic streaming velocity field is calculated numerically by solving the time-averaged second-order Navier-Stokes equation and continuity equation. ${ }^{27}$ This method only considers actuation at a single frequency, but can readily be extended to consider actuation with two frequencies by superposition of the second-order streaming flows. For this superposition to be valid the separation of the two frequencies should be much larger than the width of the resonances, which is typically on the order of $10 \mathrm{kHz}$, such that the firstorder fields of the two resonances do not couple in the timeaveraged second-order source terms for the streaming velocity field. ${ }^{28}$ However, if the two resonance frequencies are closely spaced, resulting in overlapping resonance curves, the two resonances can be excited simultaneously at a single frequency. In this case, the first-order fields of the two resonances couple in the time-averaged second-order source terms, and consequently the streaming velocity field cannot be calculated by superposition of second-order streaming flows. This case of close-lying overlapping resonances is the subject of the numerical analysis in the following section, where we show that the relative phases of the wall oscilations control the structure of the streaming flow, and that specific 
values can lead to structures qualitatively different from the standard quadrupolar Rayleigh streaming flow observed for the half-wavelength resonance. ${ }^{37}$

\section{Numerical analysis}

The following numerical analysis is a generic investigation of the acoustophoretic motion of $0.5 \mu \mathrm{m}$-diameter particles in a nearly-square channel cross section. It is not intended to be a direct numerical simulation of the actual experiments presented in this paper; nevertheless, it predicts the existence of two fundamentally different acoustic streaming patterns relevant for the interpretation of the experiments. The numerical analysis employs the method presented by Muller et al. ${ }^{28}$ and to avoid spurious effects of perfect square symmetry and to imitate the uncertainty in microchannel fabrication, the cross-sectional dimensions of the microchannel in the model was chosen to be $230.5 \mu \mathrm{m}$ wide and $229.5 \mu \mathrm{m}$ high. The parameters used in the model correspond to the biologically relevant temperature of $37^{\circ} \mathrm{C}$. The first-order velocity boundary condition applied to the walls was $u_{\mathrm{bc}}=u_{0}$ $\cos (\omega t) \boldsymbol{e}_{y}$ on the left and right walls and $u_{\mathrm{bc}}=u_{0} \cos (\omega t+\phi) \boldsymbol{e}_{z}$ on the top and bottom walls, where $u_{0}$ is the amplitude, $\omega=2 \pi f$ is the angular frequency of the transducer, $\phi$ is a constant phase shift, and $\boldsymbol{e}_{y}$ and $\boldsymbol{e}_{z}$ are the unit vectors in the transverse horizontal and vertical directions, respectively. Because both the acoustic streaming-induced drag force and the acoustic radiation force depend non-linearly on the oscillating velocity boundary conditions, the consequences of changing the phase shift $\phi$ between the two wall pairs cannot easily be deduced analytically.

To characterize the resonances of the nearly-square channel, the average acoustic energy density, ${ }^{38}$ denoted $E_{\text {ac }}$, was calculated numerically for a range of frequencies, shown in Fig. 1. This was done for several different actuations of the nearly-square channel. In each panel an inset shows a sketch of the channel geometry and which walls are actuated and by which phase factor $\cos (\omega t+\phi)$. In Fig. 1(a) the nearly-square channel was actuated in phase on the left/right walls to obtain the usual horizontal half-wavelength resonance, showing up as a Lorentzian peak centered around the resonance frequency $f_{1}=3.3032 \mathrm{MHz}$. In Fig. 1(b) the nearly-square channel was actuated in phase on the top/bottom walls resulting in a peak at the slightly higher resonance frequency $f_{2}=3.3176 \mathrm{MHz}$ corresponding to the vertical half-wavelength resonance. $f_{2}$ is slightly higher than $f_{1}$ because the height of the nearly-square channel is slightly smaller than the width. In Fig. 1(c) the nearly-square channel was actuated in phase on all four walls $(\phi=0)$. Due to the finite width of the two resonance peaks, this actuation simultaneously excites both the horizontal and the vertical half-wavelength resonances, resulting in a resonance curve with two peaks and a plateau in between, in contrast to the previous single-peak resonance curves. As a guide to the eye, the single-peak resonance curves from Fig. 1(a-b) are included in Fig. 1(c) in grey. The frequency mid-way between the two resonance peaks is

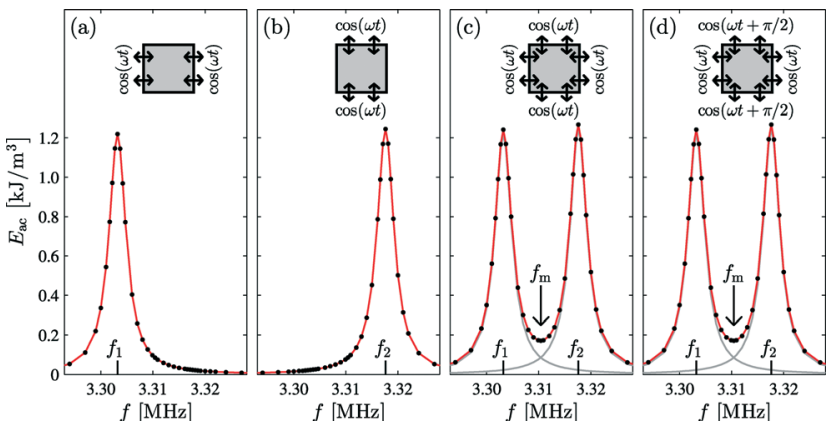

Fig. 1 Resonance curves, obtained by plotting the average acoustic energy density $E_{\mathrm{ac}} v s$. the frequency $f=\omega / 2 \pi$ of the wall actuations. (a) A nearly-square channel, $230.5 \mu \mathrm{m}$ by $229.5 \mu \mathrm{m}$ cross section, with the left/right walls vibrating in phase. (b) The nearly-square channel with the top/bottom walls vibrating in phase. (c) The nearly-square channel with all walls vibrating in phase. The resonance curves from (a) and (b) are shown in grey. (d) Same as (c) except that the top/bottom wall pair vibrate with a phase shift of $\phi=\pi / 2$ relative to the left/right wall pair. $f_{1}$ and $f_{2}$ are the two resonance frequencies corresponding to the horizontal and vertical half-wavelength resonance, respectively. $f_{m}$ indicates the middle frequency between the two resonance peaks. All walls have the same oscillation amplitude $u_{0}$.

$f_{m}=\left(f_{1}+f_{2}\right) / 2=3.3104 \mathrm{MHz}$. At this particular frequency, the amplitudes of the horizontal and the vertical resonances are the same, however much reduced relative to the two resonance maxima. In Fig. 1(d) the nearly-square channel was actuated on all four walls, but the phase of the actuation on the top/bottom wall pair was shifted relative to the left/right wall pair by $\phi=\pi / 2$. The resulting resonance curve is the same; however, as we will see below, the second-order steady acoustic streaming velocity field changes significantly by introducing this phase shift.

We now study the acoustophoretic motion of $0.5 \mu \mathrm{m}$ diameter particles in the nearly-square channel cross section, shown in Fig. 2, for each of the four actuations shown in Fig. 1. This particle motion results from the acoustic radiation force and the streaming-induced drag force, both second-order acoustic effects. ${ }^{28}$ Given the small particle diameter, the acoustophoretic motion is dominated by the drag force from the acoustic streaming. ${ }^{29}$ Fig. 2 contains four rows (a-d) corresponding to the four cases in Fig. 1. The actuation frequency was $f_{1}$ in (a), $f_{2}$ in (b), and $f_{\mathrm{m}}$ in (c-d). For each case, column 1 shows the first-order acoustic pressure, column 2 shows the acoustic radiation force together with streamlines of the steady streaming velocity field, and column 3 shows the acoustophoretic trajectories of $0.5 \mu \mathrm{m}$-diameter particles.

For the two cases $(a-b)$ the weak radiation force acts to focus the particles towards a center line, but as the particle motion is dominated by the streaming-induced drag force, they follow the quadrupolar streaming flow of the 1D halfwavelength resonance. For the two cases (c-d) both the horizontal and the vertical half-wavelength resonances are exited simultaneously at the single frequency $f_{\mathrm{m}}$, and the streaming flow is qualitatively different from the usual quadrupolar structure. For $\phi=0$ (c), the streaming flow consists of two larger 


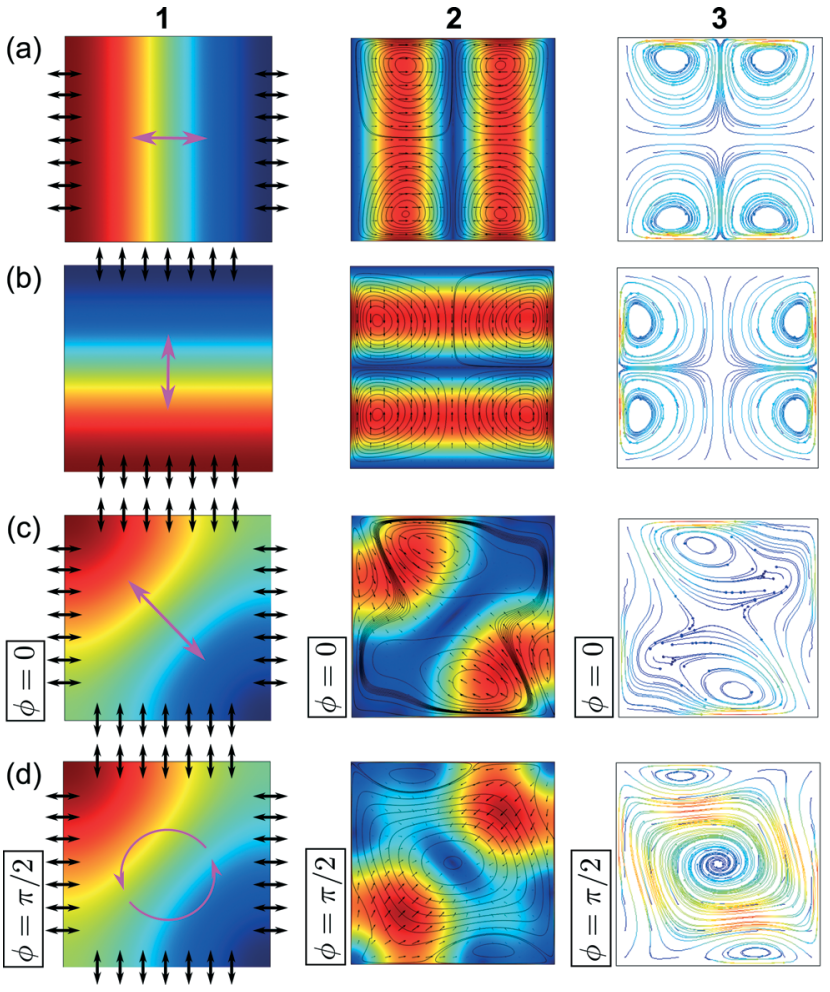

Fig. 2 Acoustophoretic motion of $0.5 \mu \mathrm{m}$-diameter particles in the nearly-square microchannel cross section. The rows (a-d) corresponds to the four cases shown in Fig. 1. The actuation frequency was $f_{1}$ in (a), $f_{2}$ in (b), and $f_{m}$ in (c-d). For each case, column 1 is a snapshot in time of the amplitude of the oscillating first-order acoustic pressure (color plot where red is positive, green is zero, and blue is negative). Column 2 is the acoustic radiation force (color plot and arrows where red is positive and blue is zero) together with streamlines (black contour lines) of the steady streaming velocity field. Column 3 is the acoustophoretic trajectories (colors indicate the speed where red is positive and blue is zero) of $0.5 \mu \mathrm{m}$-diameter particles released from a regular grid in the channel cross section. To best illustrate the qualitative results, the color scale is set by the maximum value in each plot individually. For the color plots $1(\mathrm{a}-\mathrm{c})$ the magenta arrow indicates that the pressure has an almost static nodal line (green) while the amplitude oscillates. For the color plot 1(d) the magenta arrows indicate that the nodal line (green) of the pressure field rotates in time.

flow rolls at the top and bottom walls along with two smaller flow rolls at the side walls. The small particles follow this streaming flow and are not focused in the center. For $\phi=\pi / 2$ (d), the streaming flow consists of one large flow roll in the center of the channel and two smaller flow rolls at the top and bottom walls. The combined effect of the weak radiation force towards the centre and the strong streaming-induced drag force acts to focus the particles at the centre of the channel cross section following a spiralling motion. This allows for focusing of sub-micrometer particles, which is not possible in the standard one-dimensional half-wavelength resonance $(\mathrm{a}-\mathrm{b})$. By numerically tuning the phase shift $\phi$ a solution was obtained where the large centred flow roll covered the whole channel cross section without any smaller bulk flow rolls, allowing all particles to be focused at the centre. Changing the phase shift $\phi$ by $\pi$ results in a counter- rotating streaming flow. The acoustic radiation force in Fig. 2(c-d) is similar to that reported for acoustic focusing of large particles in cylindrical channels. ${ }^{39}$ It should be stressed that the steady streaming is a boundary driven second-order flow, it is not driven by the rotation of the first-order pressure in Fig. 2(d) first column.

This numerical analysis is a generic study not aimed at direct simulation of the following experiments. Experimentally it is very difficult to control, even to measure, the vibration of the channel walls. Moreover, the wall oscillation presumably varies along the length of the channel, by analogy with what has already been verified experimentally for the acoustic field of the half-wavelength resonance. ${ }^{40} \mathrm{How}^{-}$ ever, the numerical results indicate the existence of a streaming flow that enables focusing of sub-micrometer particles, which is impossible with the well-known quadrupolar Rayleigh streaming. This new streaming flow strongly depends on the relative phase of the vibrations of the walls, i.e. the boundary conditions for the first-order acoustic field. Moreover, the spatial variation of the actuation, which has not been investigated, will presumably also influence the streaming flow and thus the focusability. This calls for a more in-depth numerical study of the dependency of the acoustic streaming on the actuation boundary condition which will be included in future work, as for the present work the main emphasis is on the experimental results.

\section{Materials and experimental methods}

\section{Design and fabrication of the device}

The chips were fabricated from $<110>$ oriented silicon using photolithography and anisotropic wet etching in $\mathrm{KOH}\left(400 \mathrm{~g} \mathrm{~L}^{-1}\right.$ $\mathrm{H}_{2} \mathrm{O}, 80^{\circ} \mathrm{C}$ ). Inlets and outlets were drilled through the silicon using a diamond drill (Tools Sverige AB, Lund, Sweden) and the chips were sealed by anodic bonding to a glass lid. The two chips had one trifurcation inlet and outlet split each, of which only a single inlet was used and the unused one was sealed (Fig. 3). The square-cross-section channel had a width

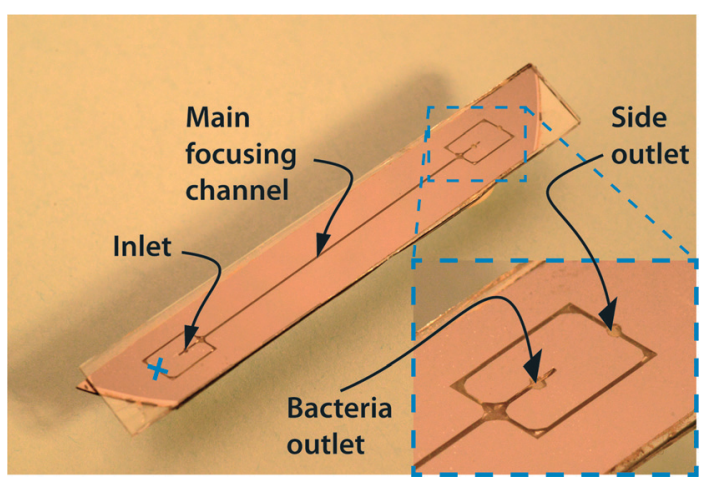

Fig. 3 Photograph of the chip design. The main focusing channel is $35 \mathrm{~mm}$ long and $230 \mu \mathrm{m}$ wide, while its height is either $230 \mu \mathrm{m}$ (square channel) or $150 \mu \mathrm{m}$ (flat rectangular channel). After the trifurcation, the side channels are connected to one outlet. The inlet marked with a blue cross is not used. 
and height of $230 \mu \mathrm{m}$ and was operated at $3.19 \mathrm{MHz}$. The rectangular-cross-section channel had a width of $230 \mu \mathrm{m}$ and a height of $150 \mu \mathrm{m}$, and was operated at $3.24 \mathrm{MHz}$ and $5.09 \mathrm{MHz}$, respectively. The piezo-ceramic ultrasound transducers (PZ26; Ferroperm piezoceramics, Kvistgaard, Denmark) actuating the chips were glued to the chips with cyanoacrylate glue (Loctite Super Glue, Henkel Norden AB, Stockholm, Sweden). The $3 \mathrm{MHz}$ ultrasound transducer was glued to the silicon and the $5 \mathrm{MHz}$ transducer was glued to the glass lid, both at the middle of the chip. To control the temperature, a Peltier element (Farnell, London, UK) was glued underneath the $3 \mathrm{MHz}$ ultrasound transducer and a Pt100 or Pt1000 resistance temperature detector (Farnell, London, UK) was glued to the glass lid.

\section{Instrument set-up}

The transducers were actuated using a dual-channel function generator (AFG 3022B; Tektronix UK Ltd., Bracknell, UK), the signals were amplified using in-house built power amplifiers based on an LT1012 power amplifier (Linear Technology Corp., Milpitas, CA, USA) and the applied voltage was monitored using an oscilloscope (TDS 2120; Tektronix). The temperature was controlled using a Peltier-controller (TC2812; Cooltronic $\mathrm{GmbH}$, Beinwil am See, Switzerland) and the temperature was set to $37^{\circ} \mathrm{C}$ throughout all experiments. Fluorescent microscopy images were obtained using a Hamamatsu camera (Hamamatsu Photonics KK, Hamamatsu, Japan) installed on an Olympus microscope (BX51WI; Olympus Corporation, Tokyo, Japan).

\section{Experimental set-up}

The flow rates were controlled using syringe pumps (neMESYS; Cetoni GmbH, Korbussen, Germany) mounted with glass syringes (Hamilton Bonaduz AG, Bonaduz, Switzerland) connected to the inlet and the outlet of the side channels. The center outlet was kept open and sample was collected from a short piece of tubing directly into an Eppendorf tube. While the inlet and outlet flow rates were varied, the outlet flow rates were kept at a split ratio of 40:60 at the center outlet and the outlet connected to the side channels. To minimize errors caused by sedimentation in the syringes and tubing, which would vary with the flow rate, sample collection with the ultrasound either on or off (for each flow rate) was compared. Particles and bacteria were quantified using a Coulter counter (Multiziser III; Beckman Coulter, Brea, CA, USA). Flow rates and voltage settings are given in Table 1.

\section{Microparticles}

Polystyrene microparticles of various sizes were used to characterize the system: $7.11 \mu \mathrm{m}, 4.99 \mu \mathrm{m}$, and $3.17 \mu \mathrm{m}$ diameter particles were obtained from Sigma-Aldrich (Buchs, Switzerland), and $0.992 \mu \mathrm{m}$ and $0.591 \mu \mathrm{m}$ particles and $0.49 \mu \mathrm{m}$ and $0.24 \mu \mathrm{m}$ fluorescent particles were obtained from Kisker (Kisker Biotech GmbH \& Co. KG, Steinfurt, Germany). Fluorescent particles $0.78 \mu \mathrm{m}$ in diameter were obtained from Bangs Laboratories (Bangs Laboratories, Fishers, IN, USA). Particle concentrations were kept below $10^{9} \mathrm{~mL}^{-1}$, to minimize the effect of acoustic and hydrodynamic interaction forces between particles.

\section{Bacteria}

For biological evaluation of the system, Escherichia coli (E. coli) DH5- $\alpha$ (containing a plasmid that carries the ampicillin-resistance gene), a kind gift from Åsa Janfalk Carlsson, was used. $E$. coli was cultured in liquid LB medium or LB plates containing $10 \mathrm{~g} \mathrm{~L}^{-1}$ tryptone (T1332; Saveen \& Werner, Limhamn, Sweden), $5 \mathrm{~g} \mathrm{~L}^{-1}$ yeast extract (Hy-Yeast 412; Sigma-Aldrich), $10 \mathrm{~g} \mathrm{~L}^{-1} \mathrm{NaCl}$ (Sigma-Aldrich) and $100 \mathrm{mg} \mathrm{L}^{-1}$ ampicillin (A9518-5G; Sigma-Aldrich) or agar (bacteriologygrade, A0949; Saveen \& Werner).

\section{Experimental results and discussion}

In what follows, a system is presented that reduces the lower particle size focusing limit for acoustophoresis to the submicrometer range, thus enabling applications in research fields such as microbiology. The experiments were carried out on two variants of an acoustophoresis microfluidic chip, which had a straight square or rectangular channel with a single inlet for particle suspensions and a trifurcation outlet split (Fig. 3). Ideally, with the onset of continuous ultrasonic actuation, particles are focused in the center of the channel and exit through the central outlet-to an extent that depends on the acoustic energy density, the flow rate of the suspension, and the size and material properties of the particles relative to the suspending liquid. In the experiments particles with diameters ranging from $0.6 \mu \mathrm{m}$ to $7 \mu \mathrm{m}$ were used and for

Table 1 Nominal flow rates $Q$ as set on the syringe pumps and voltage settings for the different experiments

\begin{tabular}{|c|c|c|c|c|c|c|c|}
\hline 5 & Sigma-Aldrich & 3.52 & $50,70,90,110,130$ & & & 4.26 & $50,60,70,8090$ \\
\hline 3 & Sigma-Aldrich & 5.72 & $70,80,90,100,150,200$ & & & 5.73 & $50,60,70,80,90$ \\
\hline 1 & Kisker & 10.4 & $10,20,30,40,50,60$ & $0-4$ & 10 & 10.6 & $15,25,35,45,55$ \\
\hline
\end{tabular}


each particle size the flow rate was varied while keeping the peak-to-peak voltage applied to the transducer constant.

In the experiments the relative focusability $R$ of the suspended particles, was measured. The relative focusability is defined as the proportion of particles moved by the ultrasound to the center outlet, particles which would otherwise end up in the side outlets if the ultrasound was not turned on. A relative focusability of $R=1$ therefore corresponds to a recovery of $100 \%$ of the particles at the center outlet while a relative focusability of $R=0$ corresponds to a recovery of $Q_{\mathrm{c}} /\left(Q_{\mathrm{c}}+Q_{\mathrm{s}}\right)=40 \%$, where $Q_{\mathrm{c}}$ and $Q_{\mathrm{s}}$ are the flow rates of the center and side outlets, respectively. The $40 \%$ recovery corresponds to the fraction of particles that would be obtained at the center outlet when the ultrasound is turned off, depending on the flow split ratio between the center and side outlets.

The transverse focusing velocity $u_{\text {rad }}$ due to the acoustic radiation force is proportional to the square of the transducer peak-to-peak voltage $U_{\mathrm{pp}}$ and the square of the particle radius a, i.e. $u_{\mathrm{rad}} \propto U_{\mathrm{pp}}{ }^{2} a^{2} .^{29}$ To be able to acquire data for different particle sizes while still maintaining a reasonable flow rate in the system, the applied voltage $U_{\mathrm{pp}}$ and therefore the acoustic energy density, was set higher in experiments involving smaller (weakly focusing) particles than in experiments with larger (strongly focusing) particles. To compare the results, the flow rates were normalized with respect to the transverse focusing velocity $u_{\mathrm{rad}}$. The $7 \mu \mathrm{m}$ particle was used as a normalization reference, as this was the largest particle used in the experiments, and it shows an almost ideal radiation force-dominated motion. The normalized flow rate $Q_{\text {norm }}$ in a particular experiments with nominal flow rates $Q$ is thus given by

$$
Q_{\text {norm }}=Q \frac{\left(u_{\mathrm{rad}}\right)_{7 \mu \mathrm{m}}}{u_{\mathrm{rad}}}=Q \frac{\left(U_{\mathrm{pp}}{ }^{2} a^{2}\right)_{7 \mu \mathrm{m}}}{U_{\mathrm{pp}}{ }^{2} a^{2}} .
$$

\section{One-dimensional focusing in a channel of rectangular cross-section}

The small size of many bioparticles such as bacteria inherently makes them less suitable for acoustic standing wave focusing in microfluidic systems without experiencing severe losses, a problem that is prominent when handling highly dilute species in situations where recoveries of more than $90 \%$ are needed.

Fig. 4(a) shows the results of one-dimensional focusing in a rectangular channel $(230 \mu \mathrm{m} \times 150 \mu \mathrm{m}$ in cross section) where relatively large polystyrene particles with diameters of $7 \mu \mathrm{m}, 5 \mu \mathrm{m}$, and $3 \mu \mathrm{m}$ (red, purple, and green) could all be focused, with a relative focusability of more than 0.9 $(R=0.98 \pm 0.10,0.93 \pm 0.003$, and $0.98 \pm 0.006$, respectively) Throughout the paper, the stated uncertainty in the value of $R$ is the standard deviation of three repeated measurements. The smaller polystyrene particles with diameters of $1 \mu \mathrm{m}$ and $0.6 \mu \mathrm{m}$ (blue and turquoise) could not be focused under the given conditions, and the focusability measured was only $R=0.52 \pm 0.17$ and $R=0.48 \pm 0.07$, respectively. For these particles, the relative focusability $R$ will not approach unity (i.e. improve) as the flow rate is decreased further because of

\section{a) Rectangular channel - 1D}

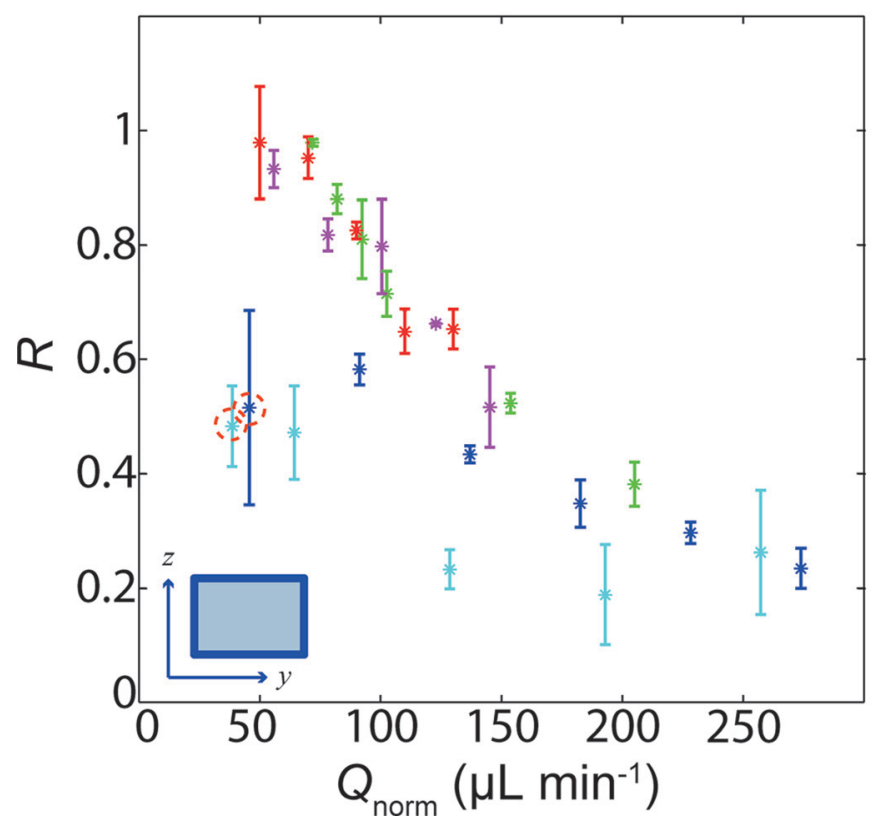

b) Square channel - 2D

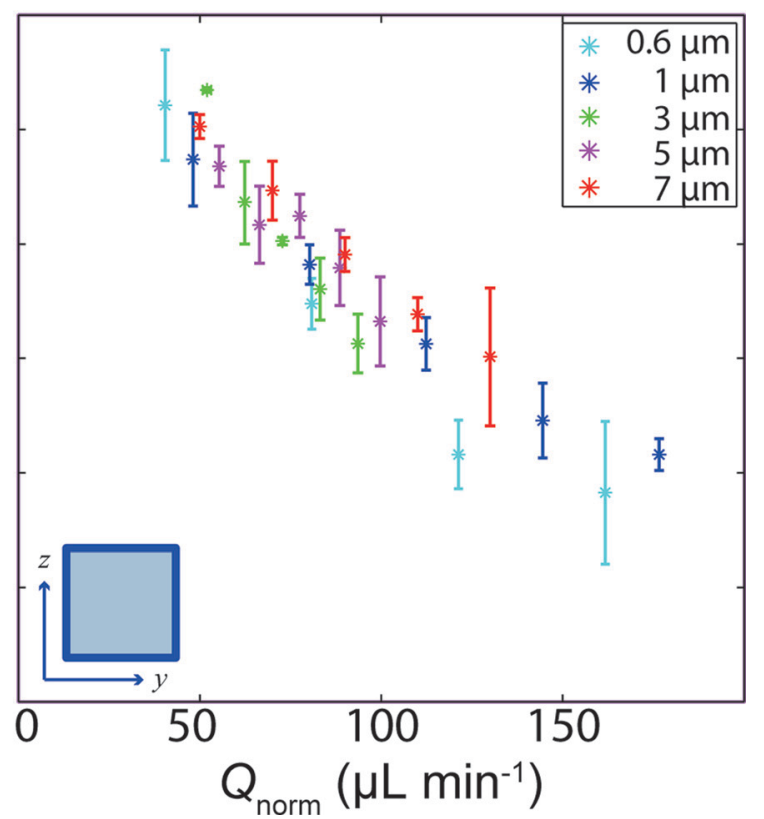

Fig. 4 (a) One-dimensional focusing in the rectangular channel. The relative focusability $R$ plotted against the normalized flow rate $Q_{\text {norm. }}$ (b) Two-dimensional single-frequency focusing in the square channel. The relative focusability $R$ plotted against $Q_{\text {norm. }}$ All error bars are standard deviations from three repeated measurements. The nominal flow rates for each data point are collected in Table 1. 
the acoustic streaming-induced drag force. This is reflected in the saturation of $R$ for the $1 \mu \mathrm{m}$ and $0.6 \mu \mathrm{m}$ particles seen for the data points obtained at the two lowest flow rates. The smaller the particle diameter, the more influence the streaming will have in comparison to the primary acoustic radiation force. This is also reflected in the fact that the focusability was generally lower for the $0.6 \mu \mathrm{m}$ diameter particles than for the $1 \mu \mathrm{m}$ diameter particles. Increasing the acoustic energy or decreasing the flow rate through the channel will not increase the focusability, since both the acoustic streaming and the acoustic radiation force depend linearly on the acoustic energy density.

For one-dimensional acoustophoretic focusing in this system, the acoustic streaming-induced drag force limits the focusability of particles less than $1.6 \mu \mathrm{m}$ in diameter. This is caused by the streaming, counteracting the radiation force in the top and bottom regions of the channel, whereby the particles are pushed outwards from the center of the channel instead of inwards. ${ }^{28}$ This can be avoided by using twodimensional focusing, without compromising the channel size or sample throughput, as presented in the following section.

\section{Two-dimensional dual-frequency focusing in a channel of rectangular cross-section}

To enable focusing of the particles in the vertical direction as well, a second piezo-ceramic ultrasound transducer was added to the rectangular channel with a half-wavelength matched to the height of the channel. This resulted in a significantly improved focusability of the $1 \mu \mathrm{m}$ and $0.6 \mu \mathrm{m}$ particles of $R=0.87 \pm 0.10$ and $R=0.92 \pm 0.34$, respectively (Fig. 5). The voltage $U_{\mathrm{pp}, 2}$ applied to the second transducer was varied at an interval from $0 \mathrm{~V}$ to $4 \mathrm{~V}$, while maintaining the settings for the flow rate and voltage of the first transducer in the corresponding one-dimensional focusing experiment at the lowest flow rate, indicated by dashed rings in Fig. 4(a). The relative focusability $R$ increased steadily as the voltage $U_{\mathrm{pp}, 2}$ approached the maximum achievable in the current system configuration. The increase in the value of $R$ for the small particles demonstrates the benefit of introducing a second orthogonal acoustic standing wave. Increasing the voltage $U_{\mathrm{pp}, 2}$ above $4 \mathrm{~V}$ may result in higher focusability, but it also caused the temperature of the system to rise above the dynamic range of the temperature regulator. An improvement in the focusability of the small particles was seen visually when the flow rate was reduced further.

\section{Two-dimensional single-frequency focusing in a channel of square cross-section}

A more straightforward way to generate two-dimensional focusing in an acoustophoresis microchannel is by using a square cross-section geometry. In this way, the same transducer operated at a single frequency can excite both the vertical and horizontal component of the standing waves. Even though the strict square symmetry is broken slightly, e.g. due to fabrication inaccuracies, the two resonances can still be

\section{Rectangular channel - 2D}

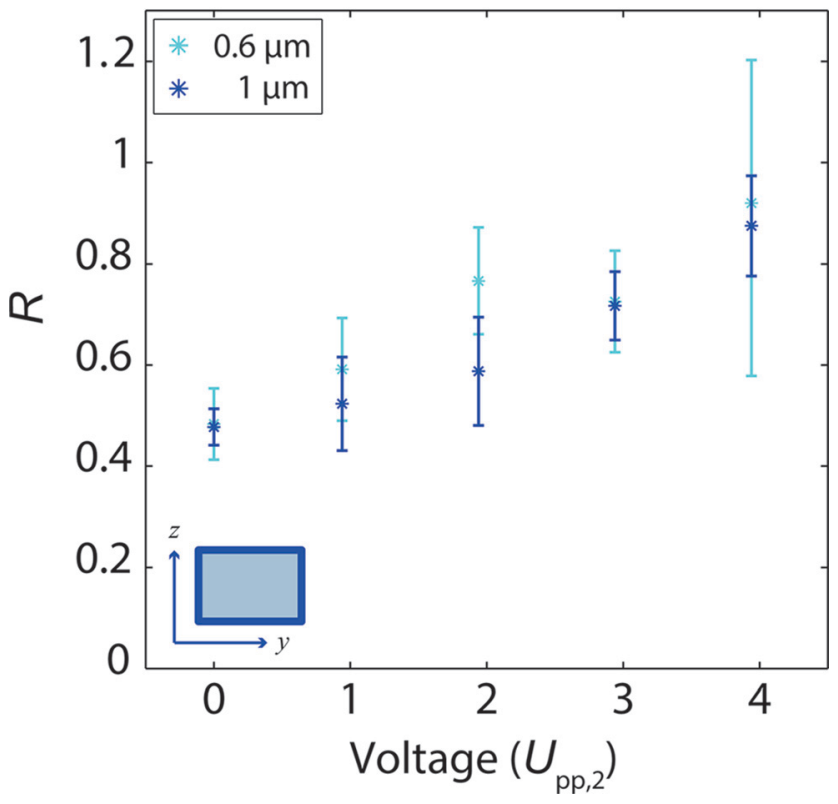

Fig. 5 Two-dimensional dual-frequency focusing in the rectangular channel. The relative focusability $R$ plotted against the voltage $U_{\mathrm{pp}, 2}$ on the second $(5 \mathrm{MHz})$ piezo transducer focusing the particles vertically in the rectangular channel. The voltage $U_{\mathrm{pp}}$ on the first $(2 \mathrm{MHz})$ transducer and the flow rate were kept constant at the same value as used to focus the particles giving the data points surrounded by the

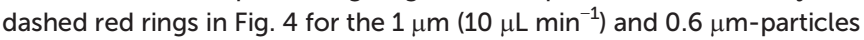
$\left(3 \mu \mathrm{L} \mathrm{min}^{-1}\right)$, respectively.

excited simultaneously due to their finite width of approximately $10 \mathrm{kHz}^{38}$

In the square channel $(230 \mu \mathrm{m} \times 230 \mu \mathrm{m}$ in cross section $)$, which supports a two-dimensional resonance, again the large particles with diameters of $7 \mu \mathrm{m}, 5 \mu \mathrm{m}$, and $3 \mu \mathrm{m}$ reached high focusability of $R=1.01 \pm 0.02,0.94 \pm 0.04$, and $1.07 \pm 0.004$, respectively (Fig. 4(b)). The smaller particles with diameters of $1 \mu \mathrm{m}$ and $0.6 \mu \mathrm{m}$ also reached high focusability $(R=0.95 \pm 0.08$ and $1.04 \pm 0.10$, respectively), thus demonstrating improved focusability compared to the one-dimensional focusing experiment. This is evident from the fact that the normalized focusability data for all the different particles now collapsed onto a single line (Fig. 4(b)) as compared to the one-dimensional focusability data (Fig. 4(a)).

The square channel cross section offers a simpler system configuration with only one frequency. In contrast, the rectangular channel required the use of two different piezoceramic transducers and therefore of two electronic driving systems (signal generators and power amplifiers), adding both cost and complexity to the system. Also, two transducers complicate the design of the temperature controller and are more likely to cause overheating, leading to a shift in frequency of the acoustic resonance and therefore poor focusing performance.

To investigate the performance of the three systems for particles less than $0.6 \mu \mathrm{m}$ in diameter, fluorescence 
microscopy was employed as these particles were too small to be quantified in the Multisizer 3 Coulter Counter used in this study. Visually, it could be observed that $0.5 \mu \mathrm{m}$ diameter fluorescent polystyrene particles could be focused in both the square (Fig. 6, Table 2) at $2 \mu \mathrm{L} \mathrm{min}^{-1}$ and the rectangular cross-section channels at $0.5 \mu \mathrm{L} \min ^{-1}$ when using two-dimensional focusing (data not shown). When using one-dimensional focusing in the rectangular cross-section channel, the particles could not be completely focused, which is consistent with our previous results. The focusability of $0.24 \mu \mathrm{m}$ fluorescent particles was also investigated, but these particles could only be seen to stream and they could not be focused in any channel (data not shown), placing the new critical particle diameter somewhere between $0.24 \mu \mathrm{m}$ and $0.5 \mu \mathrm{m}$.

\section{Bacteria focusing}

A suspension of $E$. coli was also investigated to evaluate the biological relevance of the systems. The bacteria showed a relative focusability $R=0.95 \pm 0.35$ in the square channel with two-dimensional focusing, whereas it was only $R=0.40 \pm 0.13$ in the rectangular channel using one-dimensional focusing (Table 3).

In these experiments, we deliberately kept the concentration of particles and bacteria below $10^{9} \mathrm{~mL}^{-1}$ to avoid the complication of particle-particle interaction due to hydrodynamic

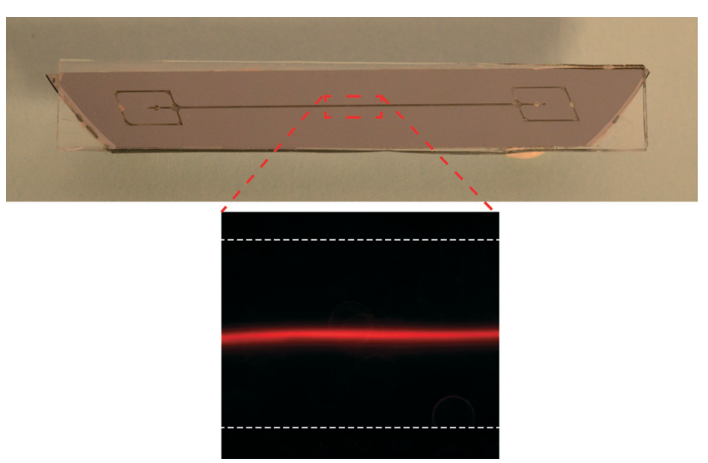

Fig. 6 Fluorescent image of $0.5 \mu \mathrm{m}$ particles (red) focusing in the square-cross-section channel at a flow rate of $2 \mu \mathrm{L} \mathrm{min}{ }^{-1}$ and at the same voltage as used for the $0.6 \mu \mathrm{m}$ particles in the square-crosssection channel focusing experiments. The broken gray lines show the edges of the channel.

Table 2 Highest relative focusability achieved for $0.5 \mu \mathrm{m}$ and $0.6 \mu \mathrm{m}$ diameter polystyrene particles

\begin{tabular}{llll}
\hline Focusing method & Particle & Relative focusability & Flow rate $Q$ \\
\hline 1D rectangle & $0.6 \mu \mathrm{m}$ & $0.48 \pm 0.07$ & $3 \mu \mathrm{L} \mathrm{min}-1$ \\
2D rectangle & $0.6 \mu \mathrm{m}$ & $0.92 \pm 0.34$ & $3 \mu \mathrm{L} \mathrm{min}$ \\
2D square & $0.6 \mu \mathrm{m}$ & $1.04 \pm 0.1$ & $5 \mu \mathrm{Lmin}^{-1}$ \\
2D square & $0.5 \mu \mathrm{m}$ & $1^{a}$ & $2 \mu \mathrm{L} \mathrm{min}$ \\
SAW $^{14, c}$ & $0.5 \mu \mathrm{m}$ & $0.79^{b}$ & $1.8 \mu \mathrm{L} \mathrm{min}^{-1}$ \\
SAW $^{15}$ & $0.5 \mu \mathrm{m}$ & $1^{a}$ & $0.2 \mu \mathrm{L} \mathrm{min}^{-1}$
\end{tabular}

${ }^{a}$ No recovery data obtained. Visual focus of particles shown.

${ }^{b}$ Recovery, no focusability data available. ${ }^{c}$ Device uses a combination of dielectrophoretic and acoustic forces.
Table 3 Highest relative focusability achievable for $E$. coli and $1 \mu \mathrm{m}$ and $0.6 \mu \mathrm{m}$ diameter polystyrene particles

\begin{tabular}{llll}
\hline Particle & 1D rectangular & 2D rectangular & 2D square \\
\hline E. coli & $0.40 \pm 0.13$ & & $0.95 \pm 0.35$ \\
$1 \mu \mathrm{m}$ & $0.52 \pm 0.17$ & $0.87 \pm 0.1$ & $0.95 \pm 0.08$ \\
$0.6 \mu \mathrm{m}$ & $0.48 \pm 0.07$ & $0.92 \pm 0.34$ & $1.04 \pm 0.1$ \\
\hline
\end{tabular}

coupling of the particles. ${ }^{41}$ Also, in future microbiological applications, the need for bacterial enrichment is most evident in samples with very low concentrations of bacteria. In contrast, previously reported focusing of $E$. coli based on a one-dimensional standing wave used a high sample concentration of $10^{10} \mathrm{~mL}^{-1}$, which caused the bacteria to agglomerate and effectively act as larger particles. ${ }^{42}$

\section{Comparison of the experimental and numerical streaming flow}

The experimental data demonstrate that sub-micrometer particles as small as $0.5 \mu \mathrm{m}$ can be focused using twodimensional acoustic focusing, which indicates that these systems are dominated by a streaming velocity field similar to that in Fig. 2(d) rather than Fig. 2(c). Importantly, the centred streaming roll derived in Fig. 2(d) has also been observed visually in some parts of the channel (video S1 of ESI $†$ ), while other parts appear to be "quieter" (i.e. not showing much streaming activity). This is consistent with the assumption that the vibration of the walls most likely changes along the channel. At different positions along the channel, the streaming rolls observed did not all move in the same direction: both clockwise and counter-clockwise streaming rolls were seen. Analogous streaming patterns have also been observed in acoustic resonance cavities with almost square geometry. ${ }^{43}$

Based on the experimental data and the numerical simulation, we hypothesise that the centred streaming roll in combination with two-dimensional focusing is the predominant effect along the full length of the channel, which enables focusing of sub-micrometer particles in the experimental square channel system presented in this paper.

\section{Conclusions}

This paper reports the successful use of acoustophoresis to focus sub-micrometer cells and particles. The use of twodimensional actuation of a square channel was found to enable two-dimensional focusing of $E$. coli and polystyrene particles as small as $0.5 \mu \mathrm{m}$ in diameter with recovery above $90 \%$, something that could not be achieved using onedimensional focusing. This sets the experimental limiting particle diameter for continuous-flow half-wavelength resonators operated at about $3 \mathrm{MHz}$ to somewhere between $0.25 \mu \mathrm{m}$ and $0.5 \mu \mathrm{m}$ for particles and bacteria with acoustic properties similar to those of polystyrene suspended in water. The focusing of sub-micrometer particles is enabled by a streaming velocity field consisting of a large centered flow roll that does not counteract the weak two-dimensional focusing, 
in contrast to the standard quadrupolar flow roll structure generated by a one-dimensional half-wavelength resonance.

The ability to manipulate bacteria and other submicrometer particles in a half-wavelength ultrasound standing wave field opens up the acoustophoresis research field to new applications in microbiology. Future research will concentrate on using the new method to generate systems capable of reducing diagnosis and detection times in the biomedical field, and in environmental and food applications.

\section{Acknowledgements}

We thank Åsa Janfalk Carlsson for the kind gift of E. coli. The work was supported by the Swedish government agency for innovation systems, Vinnova, CellCARE (grant no. 2009-00236), the Swedish Research Council (grant no. 621-2010-4389 and 2012-6708), the Royal Physiographic Society, the Crafoord Foundation, the Carl Trygger Foundation, and the Danish Council for Independent Research, Technology, and Production Sciences (grant no. 11-107021).

\section{References}

1 P. M. Dark, P. Dean and G. Warhurst, Crit. Care, 2009, 13, 217.

2 L. A. Huber, K. Pfaller and I. Vietor, Circ. Res., 2003, 92, 962-968.

3 C. Pasquali, I. Fialka and L. A. Huber, J. Chromatogr. B: Biomed. Sci. Appl., 1999, 722, 89-102.

4 C. Lay, C. Y. Teo, L. Zhu, X. L. Peh, H. M. Ji, B.-R. Chew, R. Murthy, H. H. Feng and W.-T. Liu, Lab Chip, 2008, 8, 830-833.

5 L. Zhu, Q. Zhang, H. Feng, S. Ang, F. S. Chau and W.-T. Liu, Lab Chip, 2004, 4, 337-341.

6 D. Chen and H. A. Du, Microfluid. Nanofluid., 2009, 9, 281-291.

7 B. H. Lapizco-Encinas, B. A. Simmons, E. B. Cummings and Y. Fintschenko, Anal. Chem., 2004, 76, 1571-1579.

8 S. Bhattacharya, S. Salamat, D. Morisette, P. Banada, D. Akin, Y.-S. Liu, A. K. Bhunia, M. Ladisch and R. Bashir, Lab Chip, 2008, 8, 1130-1136.

9 Z. Wu, B. Willing, J. Bjerketorp, J. K. Jansson and K. Hjort, Lab Chip, 2009, 9, 1193-1199.

10 N. Xia, T. P. Hunt, B. T. Mayers, E. Alsberg, G. M. Whitesides, R. M. Westervelt and D. E. Ingber, Biomed. Microdevices, 2006, 8, 299-308.

11 J. D. Adams, U. Kim and H. T. Soh, Proc. Natl. Acad. Sci. U. S. A., 2008, 105, 18165-18170.

12 L. R. Huang, E. C. Cox, R. H. Austin and J. C. Sturm, Science, 2004, 304, 987-990.

13 J. Shi, D. Ahmed, X. Mao, S.-C. S. Lin, A. Lawit and T. J. Huang, Lab Chip, 2009, 9, 2890-2895.

14 D. J. Collins, T. Alan and A. Neild, Lab Chip, 2014, 14, 1595-1603.

15 V. Yantchev, J. Enlund, I. Katardjiev and L. A. Johansson, J. Micromech. Microeng., 2010, 20, 035031.

16 B. Hammarström, T. Laurell and J. Nilsson, Lab Chip, 2012, 12, 4296-4304.

17 T. Laurell, F. Petersson and A. Nilsson, Chem. Soc. Rev., 2007, 36, 492-506.
18 M. Wiklund, Lab Chip, 2012, 12, 2018-2028.

19 M. A. Burguillos, C. Magnusson, M. Nordin, A. Lenshof, P. Augustsson, M. J. Hansson, E. Elmér, H. Lilja, P. Brundin, T. Laurell and T. Deierborg, PLoS One, 2013, 8, e64233.

20 J. D. Adams, C. L. Ebbesen, R. Barnkob, A. H. J. Yang, H. T. Soh and H. Bruus, J. Micromech. Microeng., 2012, 22, 075017.

21 A. Ahmad-Tajudin, K. Petersson, A. Lenshof, A.-M. Swärd-Nilsson, L. Åberg, G. Marko-Varga, J. Malm, H. Lilja and T. Laurell, Lab Chip, 2013, 13, 1790-1796.

22 F. Petersson, A. Nilsson, C. Holm, H. Jonsson and T. Laurell, Analyst, 2004, 129, 938-943.

23 A. Lenshof, A. Ahmad-Tajudin, K. Järås, A.-M. K. Swärd-Nilsson, L. Åberg, G. Marko-Varga, J. Malm, H. Lilja and T. Laurell, Anal. Chem., 2009, 81, 6030-6037.

24 C. Grenvall, P. Augustsson, J. R. Folkenberg and T. Laurell, Anal. Chem., 2009, 81, 6195-6200.

25 P. Augustsson, C. Magnusson, M. Nordin, H. Lilja and T. Laurell, Anal. Chem., 2012, 84, 7954-7962.

26 M. Nordin and T. Laurell, Lab Chip, 2012, 12, 4610-4616.

27 S. Radel, A. J. McLoughlin, L. Gherardini, O. Doblhoff-Dier and E. Benes, Ultrasonics, 2000, 38, 633-637.

28 P. B. Muller, R. Barnkob, M. J. H. Jensen and H. Bruus, Lab Chip, 2012, 12, 4617-4627.

29 R. Barnkob, P. Augustsson, T. Laurell and H. Bruus, Phys. Rev. E: Stat., Nonlinear, Soft Matter Phys., 2012, 86, 056307.

30 M. S. Limaye, J. J. Hawkes and W. T. Coakley, J. Microbiol. Methods, 1996, 27, 211-220.

31 M. Zourob, J. J. Hawkes, W. T. Coakley, B. J. Treves Brown, P. R. Fielden, M. B. McDonnell and N. J. Goddard, Anal. Chem., 2005, 77, 6163-6168.

32 R. J. Townsend, M. Hill, N. R. Harris and M. B. McDonnell, Ultrasonics, 2008, 48, 515-520.

33 O. Manneberg, J. Svennebring, H. M. Hertz and M. Wiklund, J. Micromech. Microeng., 2008, 18, 095025.

34 S. Oberti, A. Neild and J. Dual, J. Acoust. Soc. Am., 2007, 121, 778-785.

35 O. J. E. Jakobsson, C. Grenvall, M. Nordin, M. Evander and T. Laurell, Lab Chip, 2014, 14, 1943-1950.

36 M. Settnes and H. Bruus, Phys. Rev. E: Stat., Nonlinear, Soft Matter Phys., 2012, 85, 016327.

37 P. B. Muller, M. Rossi, Á. G. Marín, R. Barnkob, P. Augustsson, T. Laurell, C. J. Kähler and H. Bruus, Phys. Rev. E: Stat., Nonlinear, Soft Matter Phys., 2013, 88, 023006.

38 R. Barnkob, P. Augustsson, T. Laurell and H. Bruus, Lab Chip, 2010, 10, 563-570.

39 G. Gregory and K. Gregory, J. Acoust. Soc. Am., 2005, 117, 3440-3447.

40 P. Augustsson, R. Barnkob, S. T. Wereley, H. Bruus and T. Laurell, Lab Chip, 2011, 11, 4152-4164.

41 C. Mikkelsen and H. Bruus, Lab Chip, 2005, 5, 1293-1297.

42 J. J. Hawkes, M. S. Limaye and W. T. Coakley, J. Appl. Microbiol., 1997, 82, 39-47.

43 M. Wiklund, R. Green and M. Ohlin, Lab Chip, 2012, 12, 2438-2451. 